\title{
A longitudinal analysis of pneumococcal vaccine serotypes in pneumonia patients in Germany
}

\begin{abstract}
To the Editor:
Copyright (C)The authors 2022.

This version is distributed under the terms of the Creative Commons Attribution Non-Commercial Licence 4.0. For commercial reproduction rights and permissions contact permissions@ersnet.org

Received: 7 Sept 2021 Accepted: 1 Nov 2021

\section{○@®@}

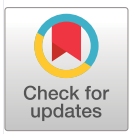

Pneumococcal infections are globally the most frequent vaccine-preventable cause of death [1], and community-acquired pneumonia (CAP) caused by Streptococcus pneumoniae is the main burden of pneumococcal disease in the elderly [2]. Since respiratory and blood cultures often remain negative in hospitalised patients with pneumococcal CAP due to prior antibiotic treatment, most cases are detected by the pneumococcal urinary antigen test (PUAT; BinaxNOW S. pneumoniae) [2, 3]. As the PUAT does not allow serotype discrimination, data on serotype distribution in adult non-bacteraemic pneumococcal CAP patients are sparse [4]. Pneumococcal conjugate vaccines (PCVs), which were primarily developed for vaccination of infants under 2 years of age, have significantly decreased invasive pneumococcal diseases worldwide in all age groups by herd protection effects [5, 6]. However, serotype replacement, i.e. replacement of vaccine serotypes by non-vaccine serotypes, has decreased the serotype coverage of PCVs over time [6, 7]. For Germany, we have described earlier the distribution of vaccine serotypes covered by the first but no longer available 7-valent pneumococcal conjugate vaccine (PCV7) and the 13-valent conjugate vaccine (PCV13) between 2002 and 2016 in adult patients with CAP enrolled into the prospective multicentre study CAPNETZ [8, 9]. PCV7 was replaced by either the 10-valent conjugate vaccine or, mainly, PCV13 in the German infant vaccination programme in 2010. However, PCV10 held the smallest market share of only 8\% of pneumococcal vaccines in Germany in 2018 [10]. In adults, the German Standing Committee on Immunization (STIKO) recommends the 23-valent pneumococcal polysaccharide vaccine (PPV23) as routine pneumococcal vaccination for all adults of 60 years and above and for all patients with defined chronic comorbidities predisposing to pneumococcal disease, regardless of age. Moreover, since 2016, sequential vaccination with PCV13 followed by PPV23 is recommended for German adults at high risk for pneumococcal disease, including individuals with immunosuppression, chronic liver disease, chronic kidney disease and individuals with cerebrospinal fluid leaks or cochlear implants [11]. Recently, a 15-valent (PCV15) and a 20-valent conjugate vaccine (PCV20) have been licensed for the adult indication by the US Food and Drug Administration and are under evaluation by the European Medicines Agency [12, 13]. PCV15 contains all serotypes of PCV13 plus serotype 22F and 33F and PCV 20 includes PCV13 serotypes plus serotypes 8, 10A, 11A, 12F, 15B, 22F and 33F.

The aim of the present study was to evaluate serotype distribution, secular trends and proportion of pneumonia caused by serotypes included in PCV13, PCV15, PCV20 and PPV23 among adult patients with all-cause CAP between 2013 and 2019. All patients enrolled in the CAPNETZ study in Germany between 1 January, 2013 and 31 December, 2019 with an available urine sample were included in the analysis. The CAPNETZ study (German Clinical Trials Register: DRKS00005274; approval number of leading ethics committee Medical Faculty of Otto-von-Guericke-University Magdeburg: 104/01; see acknowledgment or www.capnetz.de for participating centres) is a prospective observational multicentre cohort study of CAP patients treated in the hospital or in the outpatient setting. CAPNETZ inclusion criteria were age $\geqslant 18$ years, radiologically confirmed pneumonia, and at least one of the following clinical findings: cough, purulent sputum, fever or focal chest sign on auscultation. Exclusion criteria were hospitalisation during the 28 days preceding the study, immunosuppression and active tuberculosis [14]. All patients provided written informed consent prior to enrolment to the study. Urine samples of enrolled patients were prospectively collected and immediately treated with $0.5 \mathrm{M}$ 1,4-piperazinediethanesulfonic acid buffer (Boston BioProducts) to a final concentration of $25 \mathrm{mM}$ to stabilise respective polysaccharides.

\section{Shareable abstract (@ERSpublications)}

In Germany, the recently approved 20-valent pneumococcal conjugate vaccine had a substantially higher coverage against pneumonia in adults than the 13-valent vaccine, while the coverage gap compared to the 23 -valent polysaccharide vaccine was small https://bit.ly/3q4skov

Cite this article as: Bahrs C, Kesselmeier M, Kolditz M, et al. A longitudinal analysis of pneumococcal vaccine serotypes in pneumonia patients in Germany. Eur Respir J 2022; 59: 2102432 [DOI: 10.1183/ 13993003.02432-2021].
\end{abstract}


Two serotype-specific urine antigen detection (UAD) assays [15, 16] covering different serotypes on urine samples were performed and analysed at Pfizer's Vaccines Research and Development Laboratory (Pearl River, NY, USA). The UAD assay is a limit assay that uses Luminex technology, with positivity cut-off limits (based on antigen concentrations read off a standard curve), established for each serotype using 400 control urine specimens collected from otherwise healthy adults without CAP. Using nonparametric tolerance intervals, the assay is set to achieve at least $97 \%$ specificity for each serotype. UAD1 covers PCV13 serotypes [14] and UAD2 covers 11 additional serotypes (the seven included in PCV20, i.e. ST8, ST10A, ST11A, ST12F, ST15B, ST22F and ST33F, and the four included in PPV23, i.e. ST2, ST9N, ST17F and ST20) [16]. UAD analyses were performed as described previously [15, 16]. Results were classified into "positive", "indeterminate" (excluded from analysis) and "negative". According to the STIKO recommendation for pneumococcal vaccination in adults, patients were classified as "at risk for pneumococcal disease" based on age $\geqslant 60$ years or on the presence of at least one comorbidity regardless of age [11]. We quantified the distribution of pneumococcal vaccine serotypes of PCV13, PCV15, PCV20 and PPV23 as absolute and relative frequencies (relative to the number of patients with information on the respective serotype). Furthermore, we applied logistic mixed regression modelling to assess annual trends (dependent variable: each of PCV13, PCV15, PCV20, PPV23 and serotype 3; independent variable: year

TABLE 1 Distribution of pneumococcal serotypes aggregated by pneumococcal vaccine formulation in patients with radiologically confirmed community-acquired pneumonia by UAD1/UAD2 by study period and in patient subgroups with STIKO recommendation for pneumococcal vaccination (individuals $\geqslant 60$ years and individuals $18-59$ years with at-risk condition, i.e. $\geqslant 1$ comorbidity)

Overall

Study period

2013-2014 2015-2017 2018-2019

\begin{tabular}{|c|c|c|c|c|}
\hline \multicolumn{5}{|l|}{$\geqslant 18$ years of age } \\
\hline Patients & 1343 & 440 & 477 & 426 \\
\hline Any pneumococcal serotype detected by UAD1/UAD2 & $183(13.7 \%)$ & $61(13.9 \%)$ & $59(12.4 \%)$ & $63(14.9 \%)$ \\
\hline PCV13 serotypes & $103(7.7 \%)$ & $33(7.5 \%)$ & $37(7.8 \%)$ & $33(7.8 \%)$ \\
\hline PCV15 serotypes & $122(9.1 \%)$ & $43(9.8 \%)$ & $41(8.6 \%)$ & $38(9.0 \%)$ \\
\hline PCV20 serotypes & $165(12.3 \%)$ & $58(13.2 \%)$ & $51(10.7 \%)$ & $56(13.2 \%)$ \\
\hline PPV23 serotypes & $178(13.3 \%)$ & $58(13.2 \%)$ & $58(12.2 \%)$ & $62(14.7 \%)$ \\
\hline First most prevalent serotype: ST3 & $49(3.7 \%)$ & $14(3.2 \%)$ & $23(4.8 \%)$ & $12(2.8 \%)$ \\
\hline Second most prevalent serotype: ST8 & $21(1.6 \%)$ & $4(0.9 \%)$ & $5(1.1 \%)$ & $12(2.8 \%)$ \\
\hline \multicolumn{5}{|l|}{$18-59$ years with at-risk condition } \\
\hline Patients & 316 & 95 & 112 & 109 \\
\hline Any pneumococcal serotype detected by UAD1/UAD2 & $38(12.1 \%)$ & $8(8.4 \%)$ & $13(11.7 \%)$ & $17(15.6 \%)$ \\
\hline PCV13 serotypes & $23(7.3 \%)$ & $4(4.2 \%)$ & $8(7.2 \%)$ & $11(10.1 \%)$ \\
\hline PCV15 serotypes & $28(8.9 \%)$ & $5(5.3 \%)$ & $10(9.0 \%)$ & $13(11.9 \%)$ \\
\hline PCV20 serotypes & $37(11.7 \%)$ & $8(8.4 \%)$ & $13(11.7 \%)$ & $16(14.7 \%)$ \\
\hline PPV23 serotypes & $37(11.8 \%)$ & $8(8.4 \%)$ & $13(11.8 \%)$ & $16(14.7 \%)$ \\
\hline First most prevalent serotype: ST3 & $10(3.2 \%)$ & $3(3.2 \%)$ & $5(4.5 \%)$ & $2(1.8 \%)$ \\
\hline Second most prevalent serotype: ST8 & $7(2.2 \%)$ & $2(2.1 \%)$ & $2(1.8 \%)$ & $3(2.8 \%)$ \\
\hline \multicolumn{5}{|l|}{$\geqslant 60$ years of age } \\
\hline Patients & 792 & 259 & 269 & 264 \\
\hline Any pneumococcal serotype detected by UAD1/UAD2 & $113(14.4 \%)$ & $39(15.1 \%)$ & $35(13.1 \%)$ & $39(15.0 \%)$ \\
\hline PCV13 serotypes & $61(7.7 \%)$ & $19(7.3 \%)$ & $23(8.6 \%)$ & $19(7.2 \%)$ \\
\hline PCV15 serotypes & $74(9.4 \%)$ & $28(10.8 \%)$ & $25(9.3 \%)$ & $21(8.0 \%)$ \\
\hline PCV20 serotypes & $99(12.6 \%)$ & $37(14.3 \%)$ & $29(10.8 \%)$ & $33(12.6 \%)$ \\
\hline PPV23 serotypes & $110(14.0 \%)$ & $37(14.3 \%)$ & $34(12.7 \%)$ & $39(15.0 \%)$ \\
\hline First most prevalent serotype: ST3 & $33(4.2 \%)$ & $9(3.5 \%)$ & $14(5.2 \%)$ & $10(3.8 \%)$ \\
\hline Second most prevalent serotype: ST11A & $10(1.3 \%)$ & $4(1.6 \%)$ & $3(1.1 \%)$ & $3(1.1 \%)$ \\
\hline
\end{tabular}

In seven patients, more than one individual serotype was identified. Information is missing on PCV13 serotypes in two patients (one from 2017, one from 2019), on PCV15 serotypes in five patients (one from 2013, one from 2017, three from 2019), on PCV20 serotypes in five patients (one from 2016, one from 2017, three from 2019), on PPV23 serotypes in seven patients (one from 2016, two from 2017, four from 2019), on ST3 in one patient (2017), and on ST8 and on ST11A in five patients (one from 2014, one from 2015, one from 2016, two from 2019) each. Percentages refer to number of patients with available information. At-risk condition refers one or more chronic comorbidities predisposing to pneumococcal disease, as defined by STIKO. UAD: urinary antigen detection test; STIKO: German Standing Committee on Immunization; PCV: pneumococcal conjugate vaccine; PPV: pneumococcal polysaccharide vaccine; ST: serotype. 
of CAP acquisition; random effect (intercept): study centre; reported results: odds ratio with 95\% confidence interval).

Out of 1831 patients screened, urine samples with a valid UAD test result were available for 1343 patients (73.3\%) who were enrolled by 26 CAPNETZ centres distributed widely over Germany. Among these patients, 829 patients (61.7\%) were male, 792 patients (59.0\%) were aged $\geqslant 60$ years, 1038 patients (77.3\%) had at least one comorbidity, and 1204 patients (89.7\%) were treated in the hospital. Among the 1108 patients at risk for pneumococcal disease, only 179 patients (16.2\%) reported any pneumococcal vaccination within the previous 5 years. In the overall study population during the study period 2013 to 2019, 183 of 1343 (13.6\%) patients had a positive UAD1/2 test result. The most common vaccine serotypes were serotype $3(n=49 ; 3.7 \%$ of all-cause CAP), followed by serotype $8(n=21 ; 1.6 \%$ of all-cause CAP), serotype $22 \mathrm{~F}(\mathrm{n}=13 ; 1.0 \%$ of all-cause CAP) and serotype $11 \mathrm{~A}(\mathrm{n}=11 ; 0.8 \%$ of all-cause CAP). As shown in table 1 , the overall proportion of vaccine-type pneumonia among all-cause pneumonia for PCV13, PCV15, PCV20 and PPV23 was 7.7\% $(n=103), 9.1 \%(n=122), 12.3 \%(n=165)$ and $13.3 \%(n=178)$, respectively. When regarding only pneumococcal pneumonia diagnosed by conventional diagnostics (PUAT or blood culture; $\mathrm{n}=74)$, PCV13, PCV15, PCV20 and PPV23 coverage was 37.8\% $(\mathrm{n}=28), 44.6 \%(\mathrm{n}=33), 64.9 \%(\mathrm{n}=48)$ and $66.2 \%(n=49)$, respectively. Bacteraemic pneumococcal CAP was detected in $19(2.1 \%)$ of the 889 patients for whom blood cultures were obtained. Among them, bacteraemic pneumococcal CAP was caused by serotype 8 in four patients (21.1\%), serotype 4 and serotype 7F in two patients (10.5\%), and serotype 3, serotype $12 \mathrm{~F}$, serotype 14 , serotype 20 and serotype $33 \mathrm{~F}$ in one patient (5.3\%) each. The coverage of PCV13, PCV15, PCV20 and PPV23 in patients with bacteraemic CAP was $31.6 \%(n=6), 36.8 \%(n=7)$, $63.2 \%(n=12)$, and $68.4 \%(n=13)$. Over the entire observation period, we did not observe evidence for significant annual trends in pneumococcal vaccine serotype coverage (serotype 3: OR 0.95, 95\% CI $0.81-$ 1.10; PCV13: OR 0.94, 95\% CI 0.83-1.05; PCV15: OR 0.93, 95\% CI 0.84-1.03, PCV20: OR 0.95, 95\% CI 0.86-1.04; PPV23: OR 0.99, 95\% CI 0.90-1.08). Table 1 provides the serotype proportions of all-cause CAP for three time periods (2013-2014, 2015-2017 and 2018-2019) and the serotype proportion stratified by the above mentioned two STIKO classifications for patients "at risk" for pneumococcal disease (age $\geqslant 60$ years or patients $18-59$ years with $\geqslant 1$ comorbidity). Serotype 3 was the most prevalent serotype in both patient subgroups, while the second most prevalent serotype was serotype 8 in patients 18-59 years with at-risk condition and serotype $11 \mathrm{~A}$ in patients $\geqslant 60$ years.

In conclusion, PCV20 had a substantially higher coverage of all-cause CAP in adults compared to PCV13 ( $11.7 \%$ versus $7.3 \%$ for age group $18-59$ years with $\geqslant 1$ comorbidity and $12.6 \%$ versus $7.7 \%$ for age group $\geqslant 60$ years). Our data show: 1) no decline of PCV13 serotypes in all-cause CAP between 2013-2019 mainly due to a persistently high proportion of serotype 3, suggesting no meaningful effect of childhood PCV13 vaccination on PCV13 coverage in pneumonia in adults during this time period; and 2) that the gap in the coverage between PCV20 and PPV23 was small and did not increase over the entire observation time. The presented data may be of use for modelling impact of pneumococcal vaccines and may contribute to informed decision-making of vaccination committees.

\section{Christina Bahrs $\oplus^{1,2}$, Miriam Kesselmeier $\oplus^{3}$, Martin Kolditz ${ }^{4}$, Santiago Ewig ${ }^{5}$, Gernot Rohde $\oplus^{6,7,8}$, Grit Barten-Neiner $^{7,8}$, Jan Rupp ${ }^{8,9,10}$, Martin Witzenrath ${ }^{8,11}$, Tobias Welte $\overbrace{}^{7,8,12}$ and Mathias W. Pletz $\oplus^{1,8}$ for the CAPNETZ Study Group}

${ }^{1}$ Institute of Infectious Diseases and Infection Control, Jena University Hospital/Friedrich-SchillerUniversity, Jena, Germany. ${ }^{2}$ Dept of Medicine I, Division of Infectious Diseases and Tropical Medicine, Medical University of Vienna, Vienna, Austria. ${ }^{3}$ Institute of Medical Statistics, Computer and Data Sciences, Jena University Hospital/Friedrich-Schiller-University, Jena, Germany. ${ }^{4}$ Division of Pulmonology, Medical Dept I, University Hospital Carl Gustav Carus, Dresden, Germany. ${ }^{5}$ Thoraxzentrum Ruhrgebiet, Dept of Respiratory Medicine and Infectious Diseases, EVK Herne and Augusta Hospital, Bochum, Germany. ${ }^{6}$ Medical Clinic I, Dept of Respiratory Medicine, University Hospital, Frankfurt/Main, Germany. ${ }^{7}$ Biomedical Research in Endstage and Obstructive Lung Disease Hannover (BREATH), Member of the German Center for Lung Research (DZL), Hannover, Germany. ${ }^{8}$ CAPNETZ STIFTUNG, Hannover, Germany. ${ }^{9}$ Dept of Infectious Diseases and Microbiology, University Hospital Schleswig-Holstein, Lübeck, Germany. ${ }^{10}$ German Center for Infection Research (DZIF), partner site Hamburg-Lübeck-Borstel-Riems, Germany. ${ }^{11}$ Dept of Infectious Diseases and Pulmonary Medicine, and Division of Pulmonary Inflammation, Charité - Universitätsmedizin Berlin, Berlin, Germany. ${ }^{12}$ Dept of Pneumology, Hannover Medical School, Hannover, Germany. 
Acknowledgements: CAPNETZ is a multidisciplinary approach to better understand and treat patients with community-acquired pneumonia. Members of the CAPNETZ study group are: W. Knüppel (Dept of Internal Medicine, Bad Arolsen Hospital); D. Stolz (Dept of Pneumology, University Hospital Basel, Switzerland); W. Bauer (Central Emergency Admission/Medical Admission Ward, Charité-Universitätsmedizin Berlin); N. Suttorp, A. Mikolajewska and M. Witzenrath (Medical Dept, Division of Infectiology and Pneumonology Charité-Universitätsmedizin Berlin); S. Gläser and D. Thiemig (Dept of Internal Medicine - Pneumology and Infectiology, Vivantes Hospital Neukölln, Berlin-Neukölln); C. Boesecke (Medical Clinic and Policlinic I - General Internal Medicine, University Hospital Bonn); M. Prediger (III. Medical Clinic, Carl-Thiem Hospital Cottbus); B. Schaaf, J. Kremling and D. Nickoleit-Bitzenberger (Pneumology, Infectiology and Internal Intensive Care Medicine, Medical Clinic Nord, Dortmund); M. Kolditz, B. Schulte-Hubbert and S. Langner (Medical Clinic I Dept of Pneumology, University Hospital Dresden); C. Stephan (Medical Clinic II/Infectiology, University Hospital Frankfurt); G. Rohde and C. Bellinghausen (Medical Clinic I - Pneumology/Allergology, University Hospital Frankfurt); M. Panning (Institute of Virology, University Hospital Freiburg); C. Neurohr (Dept of Pneumology and Respiratory Medicine, Clinic Schillerhöhe, Gerlingen); T. Welte and I. Pink (Dept of Pneumology, Hannover Medical School, Hanover); G. Barten-Neiner, W. Kröner, M. Nawrocki and J. Naim, (CAPNETZ Office, Hannover); T. Illig and N. Klopp (Hannover Unified Biobank, Hannover Medical School); M. Pletz, B. Schleenvoigt and C. Bahrs (Institute of Infectious Diseases and Infection Control (IIMK), University Hospital Jena); C. Kroegel and A. Moeser (Clinic for Internal Medicine I, Dept of Cardiology, Angiology, Pneumology, Internistic Intensive Medicine, University Hospital Jena); D. Drömann, P. Parschke and K. Franzen (Medical Clinic III, Pneumology/Infectiology, University Medical Center Schleswig-Holstein, Lübeck); J. Rupp and N. Käding (Dept of Infectious Diseases and Microbiology, University Hospital Schleswig-Holstein, Lübeck); G. Wesseling, K. Walraven and D. Braeken (Dept of Respiratory Medicine, Maastricht University Medical Center, The Netherlands); C. Spinner (Dept of Internal Medicine II, University Hospital rechts der Isar, Technical University of Munich, School of Medicine); A. Zaruchas (Medical Clinic-Pneumology, Brüderkrankenhaus St. Josef, Paderborn); M. Falcone and G. Tiseo (Dept of Clinical and Experimental Medicine, Universita die Pisa, Italy); D. Heigener and I. Hering (Dept of Pneumology, Agaplesion Diakonieklinikum Rotenburg); W. Albrich, F. Waldeck, F. Rassouli and S. Baldesberger (Dept of Infectiology and Hospital Hygiene, Kantonsspital St. Gallen, Switzerland); S. Stenger (Institute for Medical Microbiology and Hygiene, University Hospital UIm); M. Wallner (2mt Software, Ulm); H. Burgmann, L. Traby and L. Schubert (Dept of Internal Medicine I, Division of Infectious Diseases and Tropical Medicine, Medical University of Vienna); and all study nurses.

Author contributions: All authors have made substantial contribution to the study design, data collection, analysis or interpretation, drafting the article and revising it critically for important intellectual content. All authors approved the final version to be submitted. M. Kolditz, S. Ewig, G. Rohde, G. Barten-Neiner, J. Rupp, M. Witzenrath, T. Welte and M.W. Pletz designed the study; C. Bahrs, M. Kesselmeier and M.W. Pletz drafted the article; C. Bahrs and M. Kesselmeier performed the statistical analysis; C. Bahrs, M. Kolditz, M. Kesselmeier, S. Ewig, G. Rohde, G. Barten-Neiner, J. Rupp, M. Witzenrath, T. Welte and M.W. Pletz contributed to the critical revisions, and final approval of the article.

Conflict of interest: C. Bahrs is a member of the scientific advisory board of GSK, reports personal fees from Pfizer for lectures, and has received support for attending meetings and travel, all outside the submitted work. M. Kesselmeier declares no conflict of interest. S. Ewig is member of the scientific advisory board of Pfizer. G. Rohde reports personal fees from AstraZeneca, Berlin Chemie, BMS, Pfizer, Boehringer Ingelheim, Solvay, Insmed, GSK, Essex Pharma, MSD, Grifols, Chiesi, Vertex, Roche, Takeda and Novartis for lectures including service on speakers' bureaus and/or consultancy during advisory board meetings, and personal fees from GSK for travel accommodations/meeting expenses, outside the submitted work. As part of her activity as a member of the executive bodies, G. Barten-Neiner reports economic connections to the following diagnostic and pharmaceutical companies: ThermoFisher Scientific/BRAHMS, Alere Technologies GmbH, Merck Sharp \& Dohme Corp., Pfizer Pharma GmbH, R-Biopharm AG and Helmut Hund GmbH. J. Rupp has nothing to disclose. M. Witzenrath received personal fees from AstraZeneca, Bayer Health Care, Berlin Chemie, Biotest, Chiesi, Novartis and Teva, and research funding from Actelion, Bayer Health Care, Biotest and Boehringer Ingelheim, all unrelated to the current work. T. Welte is the head of clinical studies and a member of the speakers' bureau for Pfizer, Bio Merieux and Roche Diagnostics, and is a consultant to Pfizer and MSD. M.W. Pletz is a consultant to and a member of the speakers' bureau for Bayer, MSD, Pfizer, meduptodate, Thermofisher and Novartis, and has received research grants from Pfizer.

Support statement: CAPNETZ was founded by a German Federal Ministry of Education and Research grant (01KI07145) 2001-2011. Since 2013, CAPNETZ has been supported by the German Center for Lung Research (DZL): 2013-2015 funding code 82DZL00204 and 2016-2020 funding code 82DZL002A4. C. Bahrs and M.W. Pletz are partly supported by a grant of the Federal Ministry of Education and Research KliFo 2.0 (grant number 01KI1501). M. Witzenrath is supported by grants from the German Research Foundation, SFB-TR84 C6 and C9, 
SFB 1449 B2, by the German Ministry of Education and Research (BMBF) in the framework of the CAPSyS (01ZX1304B), CAPSyS-COVID (01ZX1604B), SYMPATH (01ZX1906A), PROVID (01KI20160A) P4C (16GW0141), MAPVAP (16GW0247), NUM-NAPKON (01KX2021), and by the Berlin Institute of Health (CM-COVID). This study was supported by an unrestricted grant from Pfizer. Funding information for this article has been deposited with the Crossref Funder Registry.

\section{References}

1 Brooks LRK, Mias GI. Streptococcus pneumoniae's virulence and host immunity: aging, diagnostics, and prevention. Front Immunol 2018; 9: 1366.

2 Pletz MW, von Baum $\mathrm{H}$, van der Linden $\mathrm{M}$, et al. The burden of pneumococcal pneumonia-experience of the German competence network CAPNETZ. Pneumologie 2012; 66: 470-475.

3 Shoji $\mathrm{H}$, Domenech A, Simonetti AF, et al. The Alere BinaxNOW pneumococcal urinary antigen test: diagnostic sensitivity for adult pneumococcal pneumonia and relationship to specific serotypes. J Clin Microbiol 2018; 56: e00787-17.

4 Wunderink RG, Self WH, Anderson EJ, et al. Pneumococcal community-acquired pneumonia detected by serotype-specific urinary antigen detection assays. Clin Infect Dis 2018; 66: 1504-1510.

5 Wahl B, O'Brien KL, Greenbaum A, et al. Burden of Streptococcus pneumoniae and Haemophilus influenzae type $b$ disease in children in the era of conjugate vaccines: global, regional, and national estimates for 200015. Lancet Glob Health 2018;6: e744-e757.

6 Ladhani SN, Collins S, Djennad A, et al. Rapid increase in non-vaccine serotypes causing invasive pneumococcal disease in England and Wales, 2000-17: a prospective national observational cohort study. Lancet Infect Dis 2018; 18: 441-451.

7 Ouldali N, Varon E, Levy C, et al. Invasive pneumococcal disease incidence in children and adults in France during the pneumococcal conjugate vaccine era: an interrupted time-series analysis of data from a 17 -year national prospective surveillance study. Lancet Infect Dis 2021; 21: 137-147.

8 Pletz MW, Ewig S, Rohde G, et al. Impact of pneumococcal vaccination in children on serotype distribution in adult community-acquired pneumonia using the serotype-specific multiplex urinary antigen detection assay. Vaccine 2016; 34: 2342-2348.

9 Forstner C, Kolditz M, Kesselmeier M, et al. Pneumococcal conjugate serotype distribution and predominating role of serotype 3 in German adults with community-acquired pneumonia. Vaccine 2020; 38: 1129-1136.

10 Fortune Business Insights. Germany Pneumococcal Vaccines Market Size, Share and Industry Analysis, By Product Type (PCV10, PCV13, and PPSV23), By End User (Hospitals, Clinics, and Others) and Region Forecast, 2019-2026. www.fortunebusinessinsights.com/industry-reports/germany-pneumococcal-vaccines-market-101808 (Date last accessed: 19 Oct 2021.)

11 German Standing Committee on Vaccination at the Robert Koch Institute. Recommendation of the Standing Committee on Vaccination (STIKO) at the Robert Koch Institute - 2016/2017. Robert Koch Institut Epidemiologisches Bulletin 2016; 34: 301-337.

12 Platt HL, Greenberg D, Tapiero B, et al. A Phase II trial of safety, tolerability and immunogenicity of V114, a 15 -valent pneumococcal conjugate vaccine, compared with 13-valent pneumococcal conjugate vaccine in healthy infants. Pediatr Infect Dis J 2020; 39: 763-770.

13 Hurley D, Griffin C, Young M, et al. Safety, tolerability, and immunogenicity of a 20-valent pneumococcal conjugate vaccine (PCV20) in adults 60 to 64 years of age. Clin Infect Dis 2020; 73: e1489-e1497.

14 Welte T, Suttorp N, Marre R. CAPNETZ-community-acquired pneumonia competence network. Infection 2004; 32: $234-238$

15 Pride MW, Huijts SM, Wu K, et al. Validation of an immunodiagnostic assay for detection of 13 Streptococcus pneumoniae serotype-specific polysaccharides in human urine. Clin Vaccine Immunol 2012; 19: 1131-1141.

16 Kalina WV, Souza V, Wu K, et al. Qualification and clinical validation of an immunodiagnostic assay for detecting 11 additional Streptococcus pneumoniae serotype-specific polysaccharides in human urine. Clin Infect Dis 2020; 71: e430-e438. 